\title{
Tricuspid valve endocarditis
}

\author{
Mateo Marin-Cuartas, Martin Misfeld, Michael A. Borger, Bettina Pfannmüller \\ Leipzig Heart Center, University Department for Cardiac Surgery, Leipzig, Germany \\ Correspondence to: Bettina Pfannmüller, MD, PhD. University Department of Cardiac Surgery, Heart Center Leipzig, Struempellstrasse 39 , 04289 \\ Leipzig, Germany. Email: Bettina.pfannmueller@helios-gesundheit.de.
}

Submitted Dec 06, 2018. Accepted for publication Aug 21, 2019.

doi: $10.21037 / \mathrm{acs} .2019 .10 .02$

View this article at: http://dx.doi.org/10.21037/acs.2019.10.02

\section{Introduction}

Right-sided infective endocarditis (RSIE) encompasses $5-10 \%$ of all infective endocarditis (IE) cases. Among them, about $90 \%$ involve the tricuspid valve (TV) (1). Major risk factors for TV infective endocarditis (TVIE) are intravenous drug use, pacemaker (PM) leads and indwelling vascular accesses $(1,2)$. Only between $5-16 \%$ of RSIE cases require surgical intervention. Surgery for TVIE is indicated in the presence of TV vegetations $>20 \mathrm{~mm}$, decompensated right heart failure with severe tricuspid regurgitation (TR), recurrent pulmonary septic embolism and persistent bacteremia or sepsis due to aggressive and/or resistant microorganisms such as fungi and Staphylococcus aureus (3). Mortality of isolated TVIE is $0-15 \%$ (1). TV repair is the preferred surgical strategy; however, it is not always possible. This article presents the case of a patient with TVIE and focuses on the decision-making process and the TV operative techniques in the setting of TVIE.

\section{Clinical vignette}

A young male intravenous drug user (IVDU) without any important comorbidity presents with new onset dyspnea, fatigue and asthenia. Laboratory analysis shows an increase of inflammatory parameters. Transesophageal echocardiography demonstrates vegetations on the aortic valve and a severe aortic regurgitation (AR). Additionally, a mobile structure arising from the Eustachian valve of the inferior vena cava, a vegetation attached to the anterior leaflet of the TV and severe TR are observed. Chest computed tomography scan shows the presence of multiple pulmonary abscesses. Indication for surgery is defined by the presence of combined right and left-sided IE with severe AR and concomitant severe TR.

\section{Surgical techniques}

\section{Preparation and exposition}

TV surgery for TVIE may be performed through median sternotomy or minimally invasive through a right minithoracotomy. Minimally invasive surgery (MIS) requires full lung de-airing and collapse to allow visualization into the heart through the right mini-thoracotomy. Therefore, in patients with TVIE and multiple septic pulmonary embolisms, infarcts or abscesses, we prefer to perform median sternotomy as it allows the lung to be lightly ventilated during surgery. This reduces lung atelectasis thus facilitating an adequate postoperative mechanical ventilation and favoring early ventilator weaning and extubation. Median sternotomy is also advised in septic patients in whom a fast intervention and shorter surgery times are advocated. Regardless of the approach, the procedure is performed on total cardiopulmonary bypass. Cardiac arrest is not mandatory in the absence of atrial septal defect or patent foramen ovale thus making beating heart operations a feasible alternative.

In this case, due to the wide damage of the TV and the high complexity of the required reconstruction as well as the concomitant presence of aortic valve endocarditis with accompanying severe AR, we decided to perform a combined aortic and TV procedure through a median sternotomy, on total cardiopulmonary bypass and under cardiac arrest.

\section{Operation and completion}

Radical debridement of vegetations and infected tissue is of utmost importance and must be performed. Thereafter, the decision whether to repair or replace the TV predominantly 
depends on the extent of destruction and the repairability of the TV. If feasible, we attempt to repair the TV by means of diverse valve repair techniques depending on the regurgitation mechanism. These include TV annuloplasty, polytetrafluoroethylene (PTFE) neochord implantation by means of the loop technique (4), and autologous pericardial patch augmentation of the destroyed TV leaflets. In those patients with annular dilatation, in whom TV annuloplasty is being considered, we prefer implantation of flexible annuloplasty bands. Nevertheless, IVDUs have high recurrence rates of TVIE (1). Hence, foreign material should be avoided to reduce the risk of recurrent infection. Therefore, De Vega/Kay suture annuloplasty is preferred in IVDUs, despite being associated with high rates of recurrent TR (1). The TV is often seriously damaged and valve repair is not feasible anymore, thus making TV replacement necessary. Nonetheless, TV replacement is associated with a higher risk of recurrent TVIE in IVDUs (1).

In this case, TV repair was deemed possible. Therefore, we removed all vegetations on the anterior tricuspid leaflet as well as on the Eustachian valve. Thereafter, we excised a wide segment of the anterior tricuspid leaflet and its main chordae tendineae. Then, we performed a pericardial patch augmentation of the anterior leaflet (leaving $1 \mathrm{~cm}$ of excessive/protruding pericardial patch to increase coaptation surface) and implanted four PTFE loops. Annuloplasty was not required. Intraoperative transesophageal echocardiography confirmed a satisfactory result with only trace remaining TR.

\section{Comments}

Right mini-thoracotomy is our preferred approach, however, median sternotomy is more often performed as patients often present with contraindications for MIS. The most common contraindications are: (I) concomitant procedures such as coronary bypass surgery or aortic valve or ascending aorta replacement, (II) more than mild AR, (III) severe pleural adhesions, (IV) prominent aortic calcifications or aortic aneurysm/dissection, (V) severe peripheral artery disease, and (VI) chest wall deformity. Additionally, as mentioned above, TV related contraindications for MIS must also be considered.

PM related IE is associated with mortality rates of up to $35 \%$ (1). In these patients, we always remove the leads and the generator, as early device removal is associated with improved outcomes $(1,2)$. Insertion of new PM leads through the TV is avoided. Hence, permanent epimyocardial PM electrodes or coronary sinus leads may be alternatively implanted.

We previously published our experience in patients undergoing surgery for isolated TVIE (2). In our study 56 patients presented with isolated TVIE. Nearly $20 \%$ of patients were IVDUs, approximately $27 \%$ had a previous PM, 5.3\% had undergone implantation of contaminated devices, catheters or prostheses, and 7\% had a congenital heart defect. Median sternotomy was performed in $78.6 \%$ and right mini-thoracotomy in $21.4 \%$ of patients. The 30 -day mortality was $12.5 \%$ (6.7\% in PM patients, $0 \%$ in IVDUs, and $20 \%$ in all other patients-the latter, often with severe accompanying comorbidities). The 5 -year survival was $63.9 \%$. TV repair was performed in $60.7 \%$ of the patients, with a total of $37.5 \%$ patients who underwent isolated TV leaflet repair and $23.2 \%$ patients who underwent TV leaflet repair with additional annuloplasty (14.2\% De Vega/Kay annuloplasty and 9\% ring/band annuloplasty). TV replacement was performed in $39.3 \%$ of the patients. Among them, $30.3 \%$ received a bioprosthesis and $9 \%$ received a mechanical prosthesis. The 5 -year freedom from reoperation for recurrent TVIE was $91.7 \% \pm 4.0 \%$ (95\% CI, 152.3-179.3 months). We prefer the implantation of biological prostheses because life-long anticoagulation may be problematic, especially in patients in whom intravenous drug use is predominant and noncompliance is a major issue. Additionally, reoperation rates are similar for biological and mechanical prostheses in the tricuspid position in general population patients $(1,5)$. In patients where implantation of a prosthetic valve is not desired due to previous multiple recurrences of TVIE, tricuspid valvectomy without valve replacement may be an alternative.

Our key message is that TVIE is an uncommon entity. Almost one third of these patients are IVDUs. There are high rates of recurrent TVIE among them. Hence, not only valve competence but also a sparing use of foreign material is of utmost importance to reduce the risk of recurrent TVIE.

Finally, our patient recovered without complications and was discharged 1 week postoperatively.

\section{Acknowledgments}

None.

\section{Footnote}

Conflicts of Interest: The authors have no conflicts of interest 
to declare.

\section{References}

1. Hussain ST, Witten J, Shrestha NK, et al. Tricuspid valve endocarditis. Ann Cardiothorac Surg 2017;6:255-61.

2. Pfannmueller B, Kahmann M, Davierwala P, et al. Tricuspid Valve Surgery in Patients with Isolated Tricuspid Valve Endocarditis: Analysis of Perioperative Parameters and Long-Term Outcomes. Thorac Cardiovasc Surg 2017;65:626-33.

3. Habib G, Habib P, Antunes MJ, et al. 2015 ESC Guidelines for the management of infective endocarditis:

Cite this article as: Marin-Cuartas M, Misfeld M, Borger MA, Pfannmüller B. Tricuspid valve endocarditis. Ann Cardiothorac Surg 2019;8(6):708-710. doi: 10.21037/acs.2019.10.02
The Task Force for the Management of Infective Endocarditis of the European Society of Cardiology (ESC) Endorsed by: European Association for Cardio-Thoracic Surgery (EACTS), the European Association of Nuclear Medicine (EANM). Eur Heart J 2015;36:3075-128.

4. von Oppell UO, Mohr FW. Chordal replacement for both minimally invasive and conventional mitral valve surgery using premeasured Gore-Tex loops. Ann Thorac Surg 2000;70:2166-8.

5. Chang BC, Lim SH, Yi G, et al. Long-term clinical results of tricuspid valve replacement. Ann Thorac Surg 2006;81:1317-23, discussion 1323-4. 